\title{
Approximative Terrain Guarding With Given Number of Guards
}

\author{
Branko Kaučič \\ Faculty of Education, University of Maribor, Slovenia
}

\begin{abstract}
Guarding a surface is a well known optimization problem of the visibility site analysis and has many applications. The basic problem is searching for the minimum number of guards needed to guard (see) the entire surface. More realistic is the guarding where the number of guards is upward limited and the optimization problem is to search for their locations in order to guard as much surface as possible.

In the paper this problem is treated in detail. Several known heuristics (greedy add, greedy add with swap and stingy drop) are revised and a new technique called solution improving technique is proposed. The technique improves the results of the known algorithms and is used in indirect solving of the problem. Tests on 44 DEMs from USGS DEM Repository showed that our technique yields comparative results for smaller number of guards and better results for higher number of guards.
\end{abstract}

Keywords: optimization, terrain visibility analysis, terrain guarding, polyhedral surface, vertex-guards.

\section{Introduction}

Guarding polyhedral surfaces is known as the problem of covering/seeing a surface from a finite number of viewpoints. It combines research fields of computational geometry, combinatorial optimization and geographic information systems. It has many applications as for example: computation of optimum observation points, location of $\mathrm{TV}$, radio or mobile transmitters, communication balloons, surveillance systems such as radars, fire and watch towers. For an extensive survey about that, see [4].

Many of these applications are based on the basic guarding problem called watch tower problem [2] which searches for the minimum number of guards and their locations such that entire surface (or area of interest) is guarded (visible from guards). Because it is NP-hard [2], heuristics are used. In this paper we treat the problem which is much more realistic, but still remains NP-hard [9, 12, 4]. Specifically, instead of having unlimited number of guards, we are upward limited with their number and we are searching for the maximum guarded surface area. The problem has been addressed by several heuristics which are revised here and our new technique called solution improving technique is proposed. Proposed solution is based on indirect solving of the problem and by experimental comparison is shown that for higher number of guards our solution performs better than the known algorithms.

The paper is organized as follows. Section 2 presents the terrain model in use, guarding surface and the optimization problem. Section 3 presents known methods to solve the problem and our technique called solution improving technique. Section 4 gives experimental results where we show that our contribution yields improvements in results and Section 5 concludes the paper.

\section{Terrain Guarding}

In an abstract version of the problem, we are given a polyhedral surface, i.e. a terrain. The terrain is described by a piecewise linear continuous injective function $z=f(x, y)$ defined over a connected domain in the $X Y$ plane. In this paper, the terrain is approximated by a digital terrain model $T$ with triangular faces called a triangulated irregular network - TIN [13]. Let a TIN model describe a surface with $n_{v}$ vertices, $P_{i}=\left(x_{i}, y_{i}, z_{i}\right), i=1, \ldots, n_{v}$, and triangles 
$t_{j}, j=1, \ldots, n_{t}$. Additionally, let $A_{j}$ represent area of a surface (scalar value) for every triangle $t_{j}$. Given a viewpoint $v$ above $T$, and a generic point $p$ on $T$, the straight-line connecting $p$ to $v$ is called the line-of-sight to $p$. By definition, point $p$ is visible if and only if the line-of-sight to $p$ does not intersect the surface except at $p$. In addition, triangle $t$ is guarded from viewpoint $v$ if all its edges are visible from $v$. In such a way, a visibility function $V$ over the terrain is defined as a Boolean function, $V_{a b}=1$ if a point $P_{a}$ sees the triangle $t_{b}$, otherwise $V_{a b}=0$.

The guarding problem where we search for the minimum number of guards that cover/see the entire surface was first tackled by De Floriani et al. [3]. Several heuristics (most of them are mentioned in the next Section) are discussed by Goodchild and Lee [9], Lee [12], Franklin [8], and Eidenbenz [7]. The guarding problem is usually addressed in a discrete version, by allowing guards to be located only on the vertices (vertex-guards), or on the edges (edge-guards) of the terrain model. In special cases, as is the placement of a single guard, the algorithm running in a polynomial time is known: the guard with the lowest elevation, from which the entire terrain is guarded, can be determined in $O(n \log n)$ time on a TIN model with $n$ vertices [4]. From the theoretical point of view, significant work has been done by Bose et al. [1] and Kaučič et al. [10] who treated the problem of guarding from the worst-case optimal point of view, and Eidenbenz [7] and Eidenbenz et al. [6] by several inapproximability results.

\subsection{Guarding with Limited Number of Guards}

Restricting financial and human resources often lead to limited number of guards that are available. For example, mobile providers decide to expand their transmitter networks by $k$ new transmitters. Naturally, their goal is to maximize newly covered area by their signal.

In an abstract model of the problem we are given a terrain model and the highest possible number of guards $k$. Let the presence of guard at vertex $v_{i}$ denote a Boolean function $c, c_{i}=1$ if the guard is present, otherwise $c_{i}=0$. Then we have the following optimization problem:
Problem: given the terrain model $T$ and the visibility function $V$, find maximum guarded area by $k$ guards, that is, maximize:

$$
\sum_{j} A_{j} \min \left(1, \sum_{i} V_{i j} c_{i}\right)
$$

where

$$
\begin{aligned}
& c_{i}=\{0,1\} \quad \forall i \\
& \sum_{i} c_{i} \leq k
\end{aligned}
$$

The first constraint demands integer solution while the second constraint assures that at most $k$ guards are used. The factor $\sum_{i} V_{i j} c_{i}$ gives for a fixed triangle $t_{j}$ the number of guards that guard it. Consequently, the min gives values 1 or 0 assuring that the area of each guarded triangle is considered only once. Because the number of guards is upward limited, the surface is not necessarily entirely guarded. An example of such guarding with 5 guards covering the biggest area is shown in Fig. 1.

Note, that in the problem we search for the maximum possible area of guarded surface and not for the maximum number of triangles of the corresponding terrain model, which is not to be equalized that some heuristics in the continuation are based on triangles and not triangle areas.

The problem is not new and has been mentioned in $[9,12,4]$ as NP-hard problem. Several years back it was an important topic in Switzerland for computing the positions of communication balloons in geo-stationary orbit in order to get the maximum possible communication coverage $[5]$.

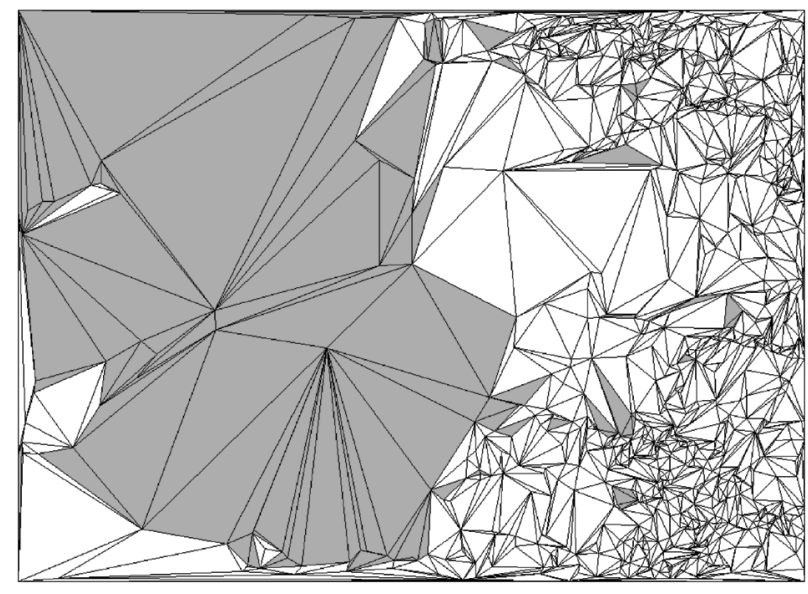

Fig. 1. An example of the biggest possible area, guarded (dark color) by 5 guards. 


\section{Heuristics}

Knowing that the problem is NP-hard is good enough reason to dismiss searching for the polynomial algorithm and rather search for the heuristic which, for the sake of speed, sacrifices exactness. In the continuation we briefly present details of three known heuristics and our new proposal - a solution improving technique. The first three heuristics have been published by Goodchild and Lee $[9,12]$ and are based on the greedy method. On the contrary, the solution improving technique was initially designed for solving the watch tower problem (see Introduction), but as it will be shown, it can be used for this problem.

Greedy add (GA) $[9,12]$ The algorithm starts with an empty solution set and then adds to this set, one at a time, the best guard (its location represented by the vertex of the terrain model). In the triangle-driven version (GAt) the best guard is the one that guards the highest number of not yet guarded triangles, while in the area-driven version (GAa) of the algorithm the best guard is the one that guards the largest area of not yet guarded triangles. The process continues until the solution set contains $k$ guards, or the entire surface is guarded.

Greedy add with swap (GAS) $[9,12]$ The GA algorithm never removes guards from the solution set. Therefore, it could be improved by including a technique that tries to improve the solution set - at each iteration the algorithm tries to replace guards, one at a time, with a guard outside the solution set. If improvement is possible, the new guard chosen to replace a particular existing guard is the one which gives the greatest improvement in the objective. However, the authors assert that searching for improvements after each newly added guard is time consuming and its usage is not justified $[9$, 12].

Stingy drop (SD) $[9,12]$ Unlike the GA and the GAS algorithms, the SD algorithm initially selects all guards (all vertices of the terrain model) into the solution set. At each step then drops the guard with the least deterioration of the objective function - the guard which covers the least areas that are visible before dropping the guard, but are invisible after dropping the guard. The process continues until the number of guards remaining in the solution set reaches $k$. Again, triangle $(\mathrm{SDt})$ - and area $(\mathrm{SDa})$ - driven approaches exist.

\subsection{Indirect Solving}

All mentioned heuristics stop their process when size of the solution set reaches number of guards $k$ (or if the entire surface is guarded). However, guarding with $k$ guards can be obtained also by first solving the watch tower problem. Let $S$ represent the solution set of the watch tower problem where whole surface is guarded. Then, in the second step, we use similar process as in the GA algorithm to select the 'best' $k$ guards, except that we select guards among solution set $S$ and not all terrain vertices.

As it is shown later in this paper, such process can yield better results. Proposed indirect solving of the problem requires solution to the watch tower problem which can be solved by modified versions of presented heuristics [9, 12]. On the contrary, in the continuation we propose our new technique for obtaining solutions with fewer guards than the presented heuristics.

Solution improving technique: The algorithms for solving the watch tower problem can be equipped with the process of searching and removing redundant guards. Namely, earlier selected guards may no longer be needed because of the covered area of the later selected guards.

Such redundant guards are clearly not needed and should be removed. They are removed from the solution set $S$, one at a time, as the 'worst' guards in the solution. The 'worst' guard is the guard guarding the smallest area, and despite its removal from the solution, the surface remains guarded. The process continues until no guard can be removed (similar as in the SD algorithm).

Removing redundant guards can be applied each time when new guard is added into the solution set or just at the end of the algorithm. Our experiments showed that applying this process to the final solution $S$, instead at each iteration of the algorithm, spent less computational time and larger number of redundant guards were removed. 


\section{Comparison of Heuristics}

The heuristics and the technique presented here were implemented in $\mathrm{C}++$ and tested on 44 actual topographic surfaces, randomly chosen from the USGS DEM repository [44]. From $1201 \times 1201$ regular gridded elevations, TIN models with $1000,2000, \ldots, 9000$ vertices were constructed by using the drop heuristic method [11]. Visibility function $V$ (see Section 2 ) for all terrain models was computed using the modified algorithm given in [12]. It was assumed that the guard were placed $1.8 \mathrm{~m}$ above terrain vertices. An example of used terrain model in its original form, Okanogan East cell, is shown in Figure 2.

Table 1 shows representative pattern of the basic elevation statistics of tested terrains and it illustrates how many guards are needed to guard (watch tower problem) the model with 3000 vertices.

Numbers of guards were computed by the watch tower problem versions of approximative algorithms GA, GAS, SD and using our solution improving technique. From the values of standard deviation of heights it can be seen that comparison included all types of terrains, flat and rough.

As mentioned in Section 3, three approaches (5 algorithms) were proposed for direct solving of the problem. Additionally, we were interested in whether using $k$ guards from the solution sets of other algorithms could be competitive (indirect solving). Algorithms were tested for three different values of number of guards: $25 \%, 50 \%$ and $75 \%$ of needed guards for the watch tower problem. Because of comprehensive results, Table 2 shows a representative pattern of the guarded area percentage of the entire surface of Alexandria West terrain cell.

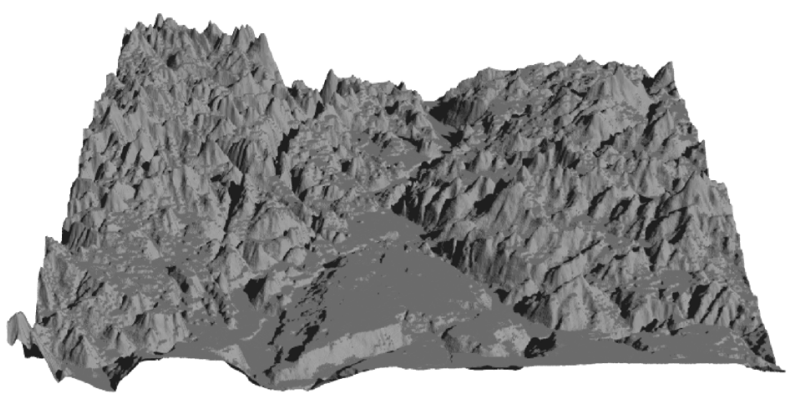

Fig. 2. An example of used terrain: Okanogan East.

\begin{tabular}{|l||r|r|r|r|r|}
\hline \multirow{2}{*}{ terrain } & \multicolumn{4}{c|}{ height } & \multirow{2}{*}{\begin{tabular}{c} 
arg \\
\cline { 2 - 5 }
\end{tabular}} \\
\cline { 2 - 5 } alexandria-w & 23 & 184 & 74,1 & 25,4 & 566 \\
ashton-w & 1513 & 3414 & 2109,4 & 319,2 & 518 \\
bend-e & 424 & 2073 & 1159,1 & 284,1 & 548 \\
caliente-e & 678 & 2865 & 1540,2 & 335,0 & 537 \\
canton-e & 201 & 461 & 337,4 & 39,3 & 628 \\
cheyenne-w & 1458 & 2926 & 2159,8 & 195,2 & 537 \\
dalhart-w & 1321 & 2657 & 1667,8 & 201,8 & 570 \\
decatur-w & 143 & 231 & 188,5 & 11,8 & 547 \\
dillon-w & 1158 & 3292 & 2080,4 & 342,2 & 568 \\
fargo-w & 274 & 464 & 349,5 & 42,2 & 494 \\
greeley-e & 1326 & 1972 & 1519,1 & 101,0 & 462 \\
lamar-e & 1014 & 1485 & 1233,9 & 87,0 & 490 \\
mariposa-w & 74 & 3962 & 1742,8 & 918,6 & 557 \\
ogden-w & 1266 & 3014 & 2012,3 & 322,5 & 569 \\
omaha-w & 291 & 475 & 373,8 & 39,4 & 600 \\
pecos-e & 668 & 1023 & 825,1 & 71,8 & 433 \\
quetico-w & 360 & 610 & 431,0 & 29,3 & 599 \\
roswell-e & 1018 & 1581 & 1214,4 & 113,6 & 521 \\
sonora-e & 499 & 791 & 686,7 & 40,9 & 581 \\
tucson-w & 378 & 1927 & 661,6 & 178,0 & 464 \\
tularosa-e & 1181 & 2704 & 1538,9 & 222,6 & 563 \\
tyler-e & 50 & 185 & 98,3 & 22,7 & 544 \\
utica-e & 98 & 1140 & 537,2 & 152,5 & 549 \\
wells-w & 1524 & 3231 & 1983,8 & 237,0 & 550 \\
wichita-w & 320 & 484 & 401,6 & 33,9 & 487 \\
\hline
\end{tabular}

Table 1. Basic elevation statistics of tested terrains and needed number of guards for the watch tower problem on model with 3000 vertices.

Results of the algorithms are divided into direct solving and indirect solving. Algorithms in indirect solving equipped with our solution improving technique are denoted by $i$ in their labels. The results of the GAS algorithm are omitted because of the known time execution problems, and the best results are emphasized. Higher value indicates better result and, based on the problem difference, $0,1 \%$ in coverage can represent a significant financial benefit. The emphasized results show that by higher number of guards our approach leads to better results (emphasized results in the line 'solution from GAai'). Omitted are the results of using the solution improving technique for the SD algorithm because our technique never found any redundant guard. 


\begin{tabular}{|l|c|ccccccccc|}
\hline algorithm & $\%$ & 1000 & 2000 & 3000 & 4000 & 5000 & 6000 & 7000 & 8000 & 9000 \\
\hline \hline \multirow{3}{*}{ GAa } & 25 & $\mathbf{7 9 . 0 2}$ & $\mathbf{7 7 . 1 9}$ & 74.63 & $\mathbf{7 5 . 6 1}$ & $\mathbf{7 6 . 5 3}$ & $\mathbf{7 8 . 3 5}$ & $\mathbf{7 7 . 9 5}$ & $\mathbf{7 7 . 8 8}$ & $\mathbf{7 7 . 7 9}$ \\
& 50 & $\mathbf{9 4 . 0 8}$ & $\mathbf{9 1 . 8 5}$ & $\mathbf{9 0 . 0 5}$ & $\mathbf{9 0 . 3 8}$ & 90.75 & 92.20 & 91.62 & 91.18 & $\mathbf{9 1 . 0 7}$ \\
& 75 & 98.02 & 95.93 & 94.46 & 94.90 & 94.98 & 96.35 & 95.68 & 95.05 & 94.97 \\
\hline \multirow{4}{*}{ GAt } & 25 & 77.53 & 75.71 & 74.37 & 74.94 & 75.74 & 77.14 & 76.74 & 77.14 & 76.90 \\
& 50 & 93.43 & 91.36 & 89.90 & 89.75 & 90.48 & 91.60 & 91.21 & 90.79 & 90.53 \\
& 75 & 97.76 & 95.95 & 94.48 & 94.75 & 95.03 & 96.26 & 95.70 & 95.00 & 94.92 \\
\hline \multirow{3}{*}{ SDa } & 25 & 75.50 & 72.11 & 72.06 & 72.55 & 73.16 & 74.88 & 74.18 & 73.92 & 74.08 \\
& 50 & 92.07 & 88.97 & 88.29 & 88.59 & 88.74 & 90.05 & 89.72 & 89.23 & 88.95 \\
SDt & 75 & 97.06 & 95.12 & 93.76 & 94.21 & 94.35 & 95.59 & 95.11 & 94.49 & 94.17 \\
\hline \hline \multirow{3}{*}{ solution from } & 25 & 77.49 & 75.96 & 73.54 & 74.53 & 75.11 & 77.09 & 76.75 & 76.83 & 76.21 \\
GAa $i$ & 50 & 92.66 & 91.27 & 89.10 & 89.68 & 89.90 & 91.25 & 90.91 & 90.38 & 89.94 \\
& 75 & 97.09 & 95.65 & 94.08 & 94.57 & 94.71 & 95.95 & 95.38 & 94.76 & 94.51 \\
\hline \multirow{3}{*}{ solution from } & 25 & 78.50 & 76.73 & 74.19 & 75.46 & 76.35 & 78.18 & 77.58 & 77.64 & 77.60 \\
GAt $i$ & 50 & 94.02 & 91.82 & 90.03 & 90.32 & $\mathbf{9 0 . 7 9}$ & $\mathbf{9 2 . 2 3}$ & $\mathbf{9 1 . 6 3}$ & $\mathbf{9 1 . 2 8}$ & 91.06 \\
& 75 & $\mathbf{9 8 . 1 1}$ & $\mathbf{9 6 . 0 6}$ & $\mathbf{9 4 . 6 2}$ & $\mathbf{9 4 . 9 8}$ & $\mathbf{9 5 . 0 8}$ & $\mathbf{9 6 . 4 0}$ & $\mathbf{9 5 . 7 5}$ & $\mathbf{9 5 . 1 7}$ & $\mathbf{9 5 . 0 4}$ \\
\hline & 50 & 76.56 & 75.48 & 74.14 & 74.62 & 75.68 & 77.03 & 76.74 & 77.03 & 76.76 \\
& 75 & 93.35 & 91.38 & 89.81 & 89.60 & 90.48 & 91.53 & 91.21 & 90.77 & 90.48 \\
& & & & & & & & & 96.01 & 94.94 \\
\hline
\end{tabular}

Table 2. Percentage of coverage from Alexandria West terrain models (higher values represent better results).

It can be also seen that $25 \%$ of needed guards (141) on average cover $77.22 \%$ of surface, $50 \%$ of needed guards (283) cover $91.48 \%$ of surface and $75 \%$ of guards (424) cover $95.69 \%$ of surface. With smaller number of guards high percentage of surface is guarded and adding extra guards yield less additional guarded surface. Consequently, an interesting question arises: how many guards are needed to achieve predefined percentage of coverage? To the author's knowledge, such problem was not posted yet.

How many times any of the algorithms and their versions, equipped with our solution improving technique, produced the best result is shown in Table 3.

The best algorithms have emphasized results. For a smaller number of guards the best algorithm is the GAa, however, it can be seen that using our solution improving technique in the GAa $i$ yielded almost one third of the best re-

\begin{tabular}{|c|cccc|}
\hline$\% \backslash$ algorithm & GAa & GAai & GAt & GAt $i$ \\
\hline \hline 25 & $\mathbf{2 6 3}$ & 127 & 3 & 2 \\
50 & 86 & $\mathbf{2 9 6}$ & 4 & 8 \\
75 & 1 & $\mathbf{3 4 6}$ & 8 & 38 \\
\hline
\end{tabular}

Table 3. How many times heuristics produced the best results. sults. As expected the results by area-driven algorithms are more suitable for solving the problem, but, surprisingly, not always so. The most important result of the testing can be seen with higher number of guards, where it is obvious that the best results are obtained with our solution improving technique (columns GAa $i$ and GAt $i$ ).

Using the indirect solving justifies also the execution times of the algorithms shown in Table 4.

Execution times of the GAS algorithm are omitted because of the known reasons (more than 3400 seconds at 3000 vertices) and the last line represents the time needed for the solution improving technique. By comparing the last line with all other lines it can be seen that our technique increases computational time by less than

\begin{tabular}{|l|ccccc|}
\hline alg. $\backslash \#$ & 1000 & 3000 & 5000 & 7000 & 9000 \\
\hline \hline GAa & 0.02 & 0.23 & 0.41 & 0.84 & 1.52 \\
GAt & 0.02 & 0.21 & 0.38 & 0.79 & 1.39 \\
SDa & 0.02 & 0.59 & 2.24 & 5.39 & 9.79 \\
SDt & 0.02 & 0.55 & 2.23 & 5.34 & 9.68 \\
\hline$i$ & 0.0009 & 0.0034 & 0.0108 & 0.0172 & 0.0312 \\
\hline
\end{tabular}

Table 4. Average execution times (in seconds) and the time needed for our solution improving technique (last line). 
$1.2 \%$ of total computational time which is remarkably little. Consequently, our technique is adequate to use when solutions are needed in short response time.

\section{Conclusion}

Visibility site analysis and terrain guarding as its special part is an important area with many applications. Typical applications are flight simulation, navigation, scenic landscape assessment, terrain exploration, military and civil surveillance, and locations of receiver/transmission facilities. Vast quantities of spatial data and increasing computational power yield even more applications.

In the paper, we treated the optimization problem of terrain guarding with upward limited number of guards, which is finding the location of pre-defined number of guards that guard as much surface as possible. Three known heuristics are revised and our technique called solution improving technique is proposed. By experimental comparison using actual terrain surfaces it is shown that for a smaller number of guards our technique yields the results comparable to the best known algorithm and for a higher number of guards it outperforms the known algorithms. In addition, an interesting question of terrain guarding is briefly proposed.

\section{References}

[1] P. Bose, T. Shermer, G. Toussaint, B. Zhu, Guarding Polyhedral Terrains. Computational Geometry Theory and Applications 1997; 7(3): pp. 173-186.

[2] R. Cole, M. ShariR, Visibility Problems for Polyhedral Terrains. Journal of Symbolic Computation 1989; 7: pp. 11-30.

[3] L. De Floriani, B. Falcidieno, C. Pienovi, D. ALLEN, G. NAGY, A visibility-based model for terrain features. Proceedings of the 2nd International Symposium on Spatial Data Handling, Seattle, 1986 Jul; pp. 235-250.

[4] L. De Floriani, E. Puppo, P. Magillo, Applications of Computational Geometry to Geographic Information Systems. Chapter 8 in Handbook of Computational Geometry, J.R. Sack, J. Urrutia (eds), Els. Sc.; 1999.
[5] S. Eidenbenz, C. Stamm, P. Widmayer, Positioning Guards at Fixed Height above a Terrain - an Optimum Inaproximability Result. Lecture Notes in Computer Science, 1461 (ESA'98), 1998; pp. 187-198.

[6] S. Eidenbenz, C. STAMm, P. WidMAYER, Inapproximability Results for Guarding Polygons and Terrains. Algorithmica, 31, Springer-Verlag NY Inc. 2001; pp. 79-113.

[7] S. EIDENBENZ, Approximation algorithms for terrain guarding. Information Proc. Letters, 82, Elsevier Science B. V. 2002; pp. 99-105.

[8] WM. R. FRANKLIN Siting observers on terrain. Joint International Symposium on Geospatial Theory, Processing and Applications - Spatial Data Handling 2002 Symposium, Ottawa, Canada 2002.

[9] M. F. GoodchILD, J. LEE, Coverage problems and visibility regions on topographic surfaces. Annals of O.R. 1989; 18: pp. 175-186.

[10] B. Kaučič, B. Žalik, F. Novak, On the lower bound of edge guards of polyhedral terrains. Int. J. Comput. Math. 2003; 80: pp. 811-814.

[11] J. LEE, A drop heuristic conversion method for extracting irregular network for digital elevation models. Proc. GIS/LIS'89, Vol. 1, Orlando, Florida, USA 1989; pp. 30-39.

[12] J. LEE, Analyses of visibility sites on topographic surfaces. Int. J. Geographical Information Systems 1991; 5(4): pp. 413-429.

[13] M. VAN KREVELD, Digital Elevation Models and TIN Algorithms. Algorithmic Foundations of Geographic Information Systems, Van Kreveld et al. (eds), LNCS tutorial, Vol. 1340, Springer; 1997: pp. 37-78.

[14] USGS

DEM, ftp://edcftp.cr.usgs.gov/pub/data/ $\mathrm{DEM} / 250$ [31/01/2004].

Recived: June, 2005 Accepted: October, 2005.

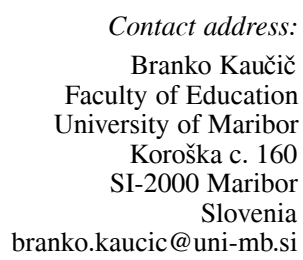

BRANKO KAUČIČ is currently a professor at Faculty of Education at the University of Ljubljana and part time teaching assistant at Faculty of Education at the University of Maribor. His primary research area are combinatorial optimization, computational geometry and geographic information systems. By merging these research fields with problems of terrain guarding he achieved his $\mathrm{PhD}$ in 2004. 